\title{
Modelado de la interconexión de los mercados de gas y electricidad en el Perú
}

\section{Modelado de la interconexión de los mercados de gas y electricidad en el Perú}

\author{
Jaime E. Luyo Kuong(*)
}

\section{RESUMEN}

En este trabajo se desarrolla un modelo económico para analizar los mercados de gas y electricidad interconectados en el Perú, considerando un ambiente oligopólico en el mercado spot de electricidad con generación hidro-térmica que interactúa con un mercado monopólico de gas donde se realizan transacciones en un mercado spot y a través de contratos forward. Se aplica un enfoque Nash-Cournot.

\section{ABSTRACT}

This paper shows an economic model to analize the interconnected gas and electricity markets in Peru, considering the spot electricity market on oligopoly environment with a hydro-thermal power generation that interacts with a monopolistic gas market that is doing spot and contracting transactions. It is applied a Nash-Cournot approach.

Palabras clave: duopolio, equilibrio Nash-Cournot, mercado spot, mercado forward.

\section{INTRODUCCIÓN}

En los Estados Unidos de Norteamérica (USA), en los meses de agosto y setiembre del 2005, los huracanes Katrina y Rita produjeron el primer impacto energético integral en el mundo, cortando en cascada el flujo de petróleo, gas natural y electricidad; reduciendo en $16 \%$ de capacidad de refinación de petróleo en USA. Además el corte energético tuvo una paralización más prolongada que si no hubiera existido interconexión entre estos sistemas energéticos; ya que los sistemas de gas y petróleo requerían que el sistema eléctrico esté funcionando para poder operar, y este último también necesitaba combustible.

En el Perú, la actual crisis energética, aunque en menor magnitud, tiene una semejanza, debido a la llegada del gas de Camisea a Lima y los incentivos de un precio subsidiado de este combustible se han instalado en los últimos años nuevos generadores termoeléctricos a gas y también ampliado la capacidad del generador dominante en el mercado eléctrico. Este nuevo escenario ha traído consigo también nuevos problemas debido a la dependencia de la generación eléctrica del suministro de gas, que ha creado una situación de crisis que se puede explicar: la crisis actual es del sector energía [1], a diferencia de la crisis del 2004 [2] que fue de insuficiencia de energía solo en el sector eléctrico, y es más grave porque es de insuficiencia de energía y de potencia en el sector eléctrico (reservas de capacidad de generación menores al 8\%, congestión en las líneas de transmisión eléctricas y de gas); y de insuficiencia de energía en el sector hidrocarburos (actual congestión del gasoducto, reservas insuficientes en el mediano plazo, precios subsidiados) que a su vez afecta el suministro energético a las generadoras

\footnotetext{
(*) Universidad Nacional de Ingeniería, Lima, Perú. Doctor en Economía, UNMSM.Master of Science in Systems and Control, Rensselaer Polytecnic Institute, USA. Postgrado en Diseño de Sistemas de Energía para el Desarrollo Sostenible, ICTP, Italia.
} 
eléctricas y además se ha suspendido la contratación de nuevos usuarios, lo que se irá complicando para el 2010 con los planes de exportación de gas a México.

Con estos antecedentes, a continuación se desarrolla un modelo teórico de los mercados de gas y electricidad en el país interconectados a través de sus respectivas redes físicas que sirven de vínculos para las transacciones. Para el actual mercado eléctrico peruano desregulado, se considera un ambiente con dos generadores dominantes (duopolio), uno hidroeléctrico y otro hidro-termoeléctrico en el mercado spot, y el resto de generadores son tomadores de precios; se aplica un enfoque Nash-Cournot. El mercado gasífero es monopólico, y las transacciones se producen en dos mercados, el spot y el de contratos forward.

La interconexión interacción de los mercados de gas y electricidad, en general se puede describir según la Figura 1.

\section{MERCADO ELÉCTRICO}

Para el caso del mercado eléctrico peruano; considerando la localización, capacidad y tipo de generación de los grupos empresariales, se formula un modelo económico estructurado como un duopolio de Cournot y una franja competitiva. El grupo empresarial de generación de producción puramente hidroeléctrica lo llamaremos empresa 1; y el grupo de producción hidro-térmoeléctrica, empresa 2; el resto de empresas conformarán la franja competitiva hidro-térmoeléctrica.

Consideraremos que cada uno de los productores tiene un reservorio integrando los reservorios individuales. El horizonte temporal, considerando el tamaño de los reservorios existentes, será mensual de corto plazo con periodos iguales en horas. También se tomará en cuenta los flujos máximos y mínimos de agua y volúmenes disponibles en los embalses expresados en unidades de energía, tanto por restricciones técnicas como por necesidades de irrigación.

En resumen, las principales características del mercado eléctrico son:

- Oligopólico [3]

- Hidro-térmico

- Concentración de Demanda en Región Centro

- Generación Termoeléctrica predominante en gas natural (Camisea)

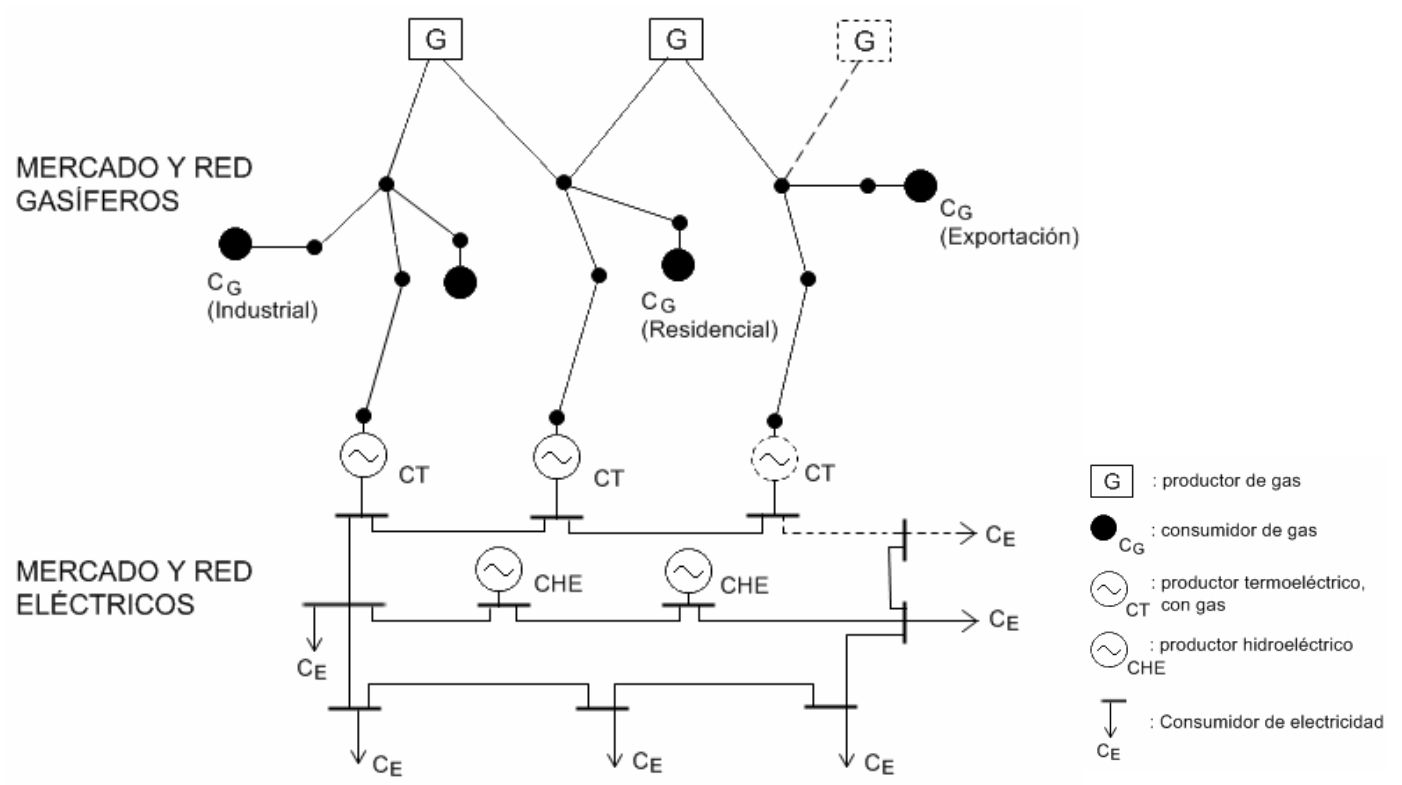

Figura 1: Interconexión de los mercados de gas y electricidad 


\section{Mercado Spot. Competencia oligopólica con enfoque Nash-Cournot}

Empresa 1 (hidroeléctrica):

$$
\begin{aligned}
& \qquad \operatorname{Max}_{q_{1 t}^{H}} \sum_{t}^{P} p_{t}\left(Q_{t}\right) \cdot q_{1 t}^{H} \\
& \text { s.a. } \quad q_{1 \min }^{H} \leq q_{1 t}^{H} \leq q_{1 \max }^{H} \quad \forall t, t=1, \ldots, P \\
& P=\text { número de períodos } \\
& q_{1 t}^{H}>0 \quad \forall t \\
& \sum_{t}^{P} q_{1 t}^{H}=\bar{q}_{1}^{H} \\
& q_{1 t}=q_{1 t}^{H}
\end{aligned}
$$

Empresa 2 (hidro-termoeléctrica):

$$
\begin{aligned}
& \underset{q_{2 t}^{H}, q_{2 t}^{T}}{\operatorname{Max}} \sum_{t}^{P} p_{t}\left(Q_{t}\right) \cdot q_{2 t}-C_{2}\left(q_{2 t}^{T}\right) \\
& \text { s.t. } \quad q_{2 \min }^{H} \leq q_{2 t}^{H} \leq q_{2 \max }^{H} \\
& q_{2 t}^{T} \leq q_{2 \max }^{T} \quad \forall t \\
& q_{2 t}^{H}, q_{2 t} \geq 0 \quad \forall t \\
& \sum_{t}^{P} q_{2 t}^{H}=\bar{q}_{2}^{H}
\end{aligned}
$$

donde:

$p_{t}\left(Q_{t}\right)=$ función inversa de demanda en el periodo $t$; precio de la electricidad.

$Q_{t}=\sum_{i=1}^{2} q_{i t}+q_{f t}+q_{M R t}=$ producción total de las empresas 1 y 2 y la franja competitiva, en el periodo $t$.

$q_{M R t}=$ producción hidroeléctrica de las empresas sin embalses (centrales de pasada), en el periodo $t$. En el caso peruano se presenta también este tipo de generación.

$q_{i t}^{H}=\quad$ producción hidroeléctrica de la empresa $i$, en el periodo $t$.

$q_{2 t}^{T}=\quad$ producción termoeléctrica de la empresa 2, en el periodo $t$.
$C_{2}\left(q_{2 t}^{T}\right)=$ función de los costos totales de las plantas térmicas de la empresa 2, en el periodo $t$.

$q_{\text {imax }}^{H}=$ máxima producción hidroeléctrica de la empresa $i$, en el periodo $t$.

$q_{i m i n}^{H}=$ mínima producción hidroeléctrica de la empresa $i$, en el periodo $t$.

$q_{2 \max }^{T}=$ máxima producción termoeléctrica de la empresa 2, en el periodo $t$.

$\bar{q}_{i}^{H}=$ máximo recurso hidroeléctrico disponible de la empresa $i$ durante todo el periodo de estudio.

$c_{2}\left(q_{2 t}^{T}\right)=$ costos marginales de la empresa 2 , en el periodo $t$.

$\sigma_{i}=\quad$ beneficio adicional de la empresa si una unidad adicional de agua se usara para la generación, que se mantiene constante durante todo el tiempo debido al horizonte temporal de corto plazo. Es el valor marginal del agua. periodos de tiempo de igual extensión $(t=1, \ldots, \mathrm{P})$ en horas, dentro de un horizonte de planificación de un mes. Se considera el mes de máxima demanda anual.

Para el máximo beneficio de las empresas generadoras con manejo estratégico (empresas 1 y 2), aplicando las condiciones de Karush-Kuhn-Tucker considerando además que los generadores no alcanzan a tener restricciones de capacidad, los ingresos marginales de las empresas 1 y 2 resultan:

$$
\begin{array}{ll}
I M_{1 t}=p_{t}\left(Q_{t}\right)+\frac{\partial p_{t}}{\partial Q_{t}} \cdot q_{1 t}^{H}=\sigma_{1} & \forall t \\
I M_{2 t}=p_{t}\left(Q_{t}\right)+\frac{\partial p_{t}}{\partial Q_{t}} \cdot q_{2 t}=c_{2}\left(q_{2 t}^{T}\right) & \forall t \\
I M_{2 t}=p_{t}\left(Q_{t}\right)+\frac{\partial p_{t}}{\partial Q_{t}} \cdot q_{2 t}=\sigma_{2} & \forall t
\end{array}
$$

y para la franja competitiva (donde están incluidas las otras empresas hidroeléctricas y termoeléctricas a gas, que no tienen comportamiento estratégico ${ }^{1}$ ); el costo

${ }^{1}$ además las más grandes están operando como generadoras de base 
marginal y valor marginal del agua equivalentes resultan iguales al precio del mercado.

$$
\begin{array}{ll}
p_{t}=c_{f}\left(q_{f t}^{T}\right) & \forall t \\
p_{t}=\sigma_{f} & \forall t
\end{array}
$$

considerando que la demanda residual del mercado es:

$D\left(p_{t}\right)=A_{t}-B \cdot p_{t} \quad, \forall t, t=1, \ldots, P$

y el costo marginal de la producción térmica del generador 2:

$c_{2}\left(q_{2 t}^{T}\right)=K_{2}+m_{2} q_{2 t}^{T}$

La solución de equilibrio de Nash-Cournot, permite determinar que la producción térmica a la empresa dominante 2 está aplicando withholding a lo largo del periodo de operación de máxima demanda anual cuya expresión es:

$$
q_{2 t}^{T}=\frac{\sigma_{2}-K_{2}}{m_{2}}, \quad \forall t
$$

$t=$ periodo

$\sigma_{2}=$ valor marginal del agua en el periodo considerado, y que se mantiene constante en el corto plazo. Para mayor horizonte de tiempo se considera variable en el tiempo, $\sigma_{2 t}$

\section{MERCADO GASÍFERO}

El mercado del gas en el Perú, a partir del 2004 cuando se inició el transporte a Lima, ha tenido un crecimiento de la demanda que superó largamente su proyección inicial, ya que actualmente existe una crisis de oferta debido a la congestión del gasoducto Camisea-Lima, y está además en desarrollo un proyecto de exportación a través de un ramal hacia el puerto de Pisco. En el modelado siguiente, hacemos abstracción de aspectos coyunturales, considerando un escenario mediato donde existe suficiente oferta y una demanda satisfecha; es decir se han confirmado nuevas reservas de gas atendiéndose el mercado interno como el externo. Se asume que las pérdidas de transporte son despreciables y que el precio del gas no está subsidiado.
El mercado gasífero actual está caracterizado por:

- Producción y transporte monopólico (y con el mismo propietario mayoritario);

- Actualmente existe un gaseoducto Camisea-Lima que alimenta la generación termoeléctrica localizada en Lima, y un ramal a Pisco para la exportación;

- Existe un mercado spoty uno de contratos forward [4]

Los mercados de gas y electricidad se interconectan. En el corto plazo, la parte importante y mayor de la demanda de gas está en el consumo de los generadores termoeléctricos, y el mayor consumidor es el de la empresa 2, $\left(q_{2 t}^{T}\right)$, y la industria localizada en la costa de Lima; y en el mediano plazo la demanda crecerá mayormente en el componente variable de la demanda, $D_{I_{t}}$, al desarrollarse el mercado al mediano plazo.

\section{Demanda de Gas}

Está conformada por:

$D_{G_{t}}=\left(\eta_{2} q_{2 t}^{T}+\eta_{e} q_{e t}^{T}\right)+D_{I_{t}}, \quad \forall t$

$D_{I_{t}}=$ demanda de exportación + industria + residencial, en el periodo $t$

$\eta_{e}, \eta_{2}=$ factor de conversión $\mathrm{x}$ eficiencia ${ }^{-1}$ equivalente (otras generadoras), y generadora 2

Se asume que: $D_{I_{t}}=a_{t}-b \cdot p_{G_{t}}$,

$\therefore \quad D_{G_{t}}=\left(\eta_{2} q_{2 t}^{T}+\eta_{e} q_{e t}^{T}\right)+a_{t}-b \cdot p_{G_{t}} \quad \forall t$

$p_{G_{t}}=$ precio del gas en el periodo $\mathrm{t}$

$q_{G_{t}}=$ capacidad de producción de gas (CF/ hora), en el periodo $t$

$q_{e t}^{T}=$ capacidad de generación eléctrica de empresa equivalente no estratégica ${ }^{2}$, en el periodo $t$

Asumiendo que no hay pérdidas ni retraso en el transporte: $q_{G_{t}}=D_{G_{t}}$ además $\eta_{e} q_{e t}^{T}$ está incorporada en $a_{t}$, y $\eta_{2}=\eta$.

\footnotetext{
2 Actualmente se observa que en el SEIN, las termoeléctricas están produciendo incluso en la base de la curva de duración de carga [5][6], por lo tanto es consistente considerar que su demanda de gas es intensa y constante en el tiempo. Esto, con el comportamiento de withholding de la empresa 2; permite asumir consistentemente que la demanda de gas es muy inelástica en el corto plazo, aumentando su elasticidad en el mediano plazo.
} 


\section{Mercado Spot}

Se considera que la productora gasífera ha realizado contratos forward en una cantidad

$q_{G_{t}}^{f}$ y a un precio acordado $s_{t}$ (strike price);

su beneficio a lo largo del tiempo es:

$\Pi_{G}^{S}=\sum_{t} p_{G_{t}} \cdot\left(q_{G_{t}}-q_{G_{t}}^{f}\right)-C\left(q_{G_{t}}\right) \quad, \forall t$

Se considera que:

$C\left(q_{G_{t}}\right)=K \cdot q_{G_{t}}+\frac{m}{2} q_{G_{t}}^{2} \quad, \forall t$

Para max. $\Pi_{G}^{S}$

$$
\begin{aligned}
& p_{G_{t}}+\left(q_{G_{t}}-q_{G_{t}}^{f}\right) p_{G_{t}}^{\prime}-K-m q_{G_{t}}=0 \\
& \therefore \quad q_{G_{t}}=\frac{\eta \cdot q_{2_{t}}^{T}+a_{t}+q_{G_{t}}^{f}-b \cdot K}{(2+b \cdot m)}, \forall t \\
& p_{G_{t}}=\frac{(1+b \cdot m) \eta \cdot q_{2_{t}}^{T}+a_{t}(1+b \cdot m)-q_{G_{t}}^{f}+b \cdot K}{(2+b \cdot m) b}, \forall t
\end{aligned}
$$

\section{Mercado Forward}

El beneficio de la empresa gasífera tomando en cuenta el ingreso intertemporal del mercado spot:

$$
\begin{array}{r}
\Pi^{f}=\sum_{t} s_{t} \cdot q_{G_{t}}^{f}+\delta \cdot \sum_{t} p_{G_{t}} \cdot\left(q_{G_{t}}-q_{G_{t}}^{f}\right) \\
-C\left(q_{G_{t}}\right) \\
\forall t ; \delta
\end{array}
$$

(factor de descuento) $<1$

$$
\Pi^{f}=\underbrace{\sum_{t} \delta\left[p_{G_{t}} \cdot q_{G_{t}}-\left(K \cdot q_{G_{t}}+\frac{m}{2} q_{G_{t}}^{2}\right)\right.}_{\text {beneficios actualizados }}]+\underbrace{\left[s_{t}-\delta \cdot p_{G_{t}}\right]_{G_{t}}^{f}}_{\begin{array}{c}
\text { benef. de } \\
\text { arbitraje }
\end{array}}
$$

la presencia de especuladores elimina la posibilidad de arbitraje; $s_{t}=\delta \cdot p_{G_{t}}$

para Max $\Pi^{f}$ :

$$
p_{G_{t}}\left(\frac{1}{2+b \cdot m}\right)+q_{G_{t}} \frac{(-1)}{b(2+b \cdot m)}-\frac{K+m \cdot q_{G_{t}}}{2+b \cdot m}=0
$$

Considerando los resultados anteriores y, resolviendo para máximo beneficio total

$$
q_{G_{t}}^{f} \rightarrow 0 \quad, \quad \forall t
$$

además

$$
S_{t}=\delta \cdot \frac{(1+b \cdot m) \eta \cdot q_{2_{t}}^{T}+a_{t}(1+b \cdot m)-q_{G_{t}}^{f}+b \cdot K}{(2+b \cdot m) b} \quad, \forall t
$$

\section{CONCLUSIONES}

- Se ha desarrollado un modelo de equilibrio dinámico de la interconexión de los mercados de gas y electricidad en Perú.

- El precio del gas en el mercado gasífero está determinado en el corto plazo ${ }^{3}$ por la generación térmica de la empresa dominante en el mercado de electricidad. También está en función de la variación de la demanda de gas en el tiempo.

- El nivel del precio del gas es mitigado (reducción del poder de mercado) en el mercado spot y mercado forward por la magnitud de la capacidad transada en el mercado de contratos de gas en cualquier periodo de tiempo.

- El precio es más elevado cuando la demanda en el mercado (spot y forward) de gas es más inelástica, en el corto plazo. A mediano plazo, si el regulador promueve la competencia en la actividad de comercialización minorista donde actualmente se observa colusión tácita (o concertada) donde el margen del comercializador del GLP llega al 50\%, entonces la elasticidad-precio de la demanda será mayor influyendo en la reducción del precio del gas. Situación análoga a la observada actualmente en el mercado de electricidad.

- Cuando no hay oportunidad de arbitraje, el monopolista maximiza sus beneficios reduciendo al mínimo el volumen de contratos; es decir, realizando sus transacciones básicamente en el mercado spot.

\footnotetext{
3 Actualmente, la demanda de gas en los generadores eléctricos se estima alrededor del $70 \%$
} 


\section{REFERENCIAS BIBLIOGRÁFICAS}

[1] Luyo, Jaime E. Competencia, Seguridad y Sostenibilidad en el Mercado Eléctrico Peruano, XVII Reunión del Congreso Nacional de Ingeniería Mecánica, Eléctrica y Ramas Afines (CONIMERA), Lima, 05 de julio del 2007.

[2] Luyo, Jaime E. Reforma del Sector Eléctrico en Perú: La Crisis del 2004 y Cambios Necesarios, Revista de la Facultad de Ciencias Económicas, UNMSM, en edición septiembre/octubre 2006.

[3] Luyo, Jaime E. Crisis del Sector Eléctrico en Perú: Liberalización y Poder de Mercado. Aplicación del MCP, International Conference on Mathematics, Instituto de Matemática y Ciencias Afines, IMCA-UNI, Lima 06 de diciembre del 2006.

[4] Neuhoff, Karsten, and Christian von Hirshhausen, Long-Term vs. Short-Term Contracts: A European Perspective on Natural Gas, University of Cambridge, Faculty of Economics, july 2005.

[5] Operación Económica del Sistema (COES). www.coes.org.pe.

[6] Osinerg. www.osinerg.org.pe.

[7] Ministerio de Energía y Minas. www.minem.gob.pe.

E-mail: jeluyo@yahoo.es 